\title{
Boundary behaviors for a class of continuous-state nonlinear branching processes in critical cases
}

\author{
Shaojuan $\mathrm{Ma}^{*} \quad \mathrm{Xu}$ Yang $^{\dagger} \quad$ Xiaowen $\mathrm{Zhou}^{\ddagger}$
}

\begin{abstract}
Using Foster-Lyapunov techniques we establish new conditions on non-extinction, non-explosion, coming down from infinity and staying infinite, respectively, for the general continuous-state nonlinear branching processes introduced in Li et al. (2019). These results can be applied to identify boundary behaviors for the critical cases of the above nonlinear branching processes with power rate functions driven by Brownian motion and (or) stable Poisson random measure, which was left open in Li et al. (2019). In particular, we show that even in the critical cases, a phase transition happens between coming down from infinity and staying infinite.
\end{abstract}

Keywords: continuous-state branching process; nonlinear branching; extinction; explosion; come down from infinity; stay infinite; Foster-Lyapunov criteria; stochastic differential equation. MSC2020 subject classifications: $60 \mathrm{~F} 10 ; 60 \mathrm{C} 05$.

Submitted to ECP on September 24, 2020, final version accepted on January 24, 2021.

\section{Introduction}

Continuous-state branching processes (CSBPs for short) are nonnegative-valued Markov processes satisfying the additive branching processes. They often arise as timepopulation scaling limits of discrete-state branching processes, and can also be obtained from spectrally positive Lévy processes via the Lamperti transform. The introduction of CSBP allows the applications of stochastic analysis, Lévy processes and stochastic differential equations (SDEs for short) techniques to its study. We refer to Li [12], Li [13] and Kyprianou [4] and references therein for comprehensive reviews on CSBPs.

Generalized versions of the CSBP have been proposed in recent years to incorporate interactions between individuals and (or) between individuals and the population. A class of CSBPs with nonlinear branching mechanism, obtained by generalized Lamperti transform, is introduced in Li [9]. In Li et al. [11], a more general version of the nonlinear CSBP is proposed as the solution to SDE

$$
X_{t}=x+\int_{0}^{t} a_{0}\left(X_{s}\right) \mathrm{d} s+\int_{0}^{t} \int_{0}^{a_{1}\left(X_{s}\right)} W(\mathrm{~d} s, \mathrm{~d} u)+\int_{0}^{t} \int_{(0, \infty)} \int_{0}^{a_{2}\left(X_{s-}\right)} z \tilde{M}(\mathrm{~d} s, \mathrm{~d} z, \mathrm{~d} u)
$$

${ }^{*}$ School of Mathematics and Information Science, North Minzu University, China. E-mail: sjma@nmu.edu.cn

${ }^{\dagger}$ School of Mathematics and Information Science, North Minzu University, China. Corresponding author. E-mail: xuyang@mail.bnu.edu.cn

${ }^{\ddagger}$ Department of Mathematics and Statistics, Concordia University, Canada. E-mail: xiaowen.zhou@ concordia.ca 
where $x>0, a_{0}$ and $a_{1}, a_{2} \geq 0$ are Borel functions on $[0, \infty), W(\mathrm{~d} s, \mathrm{~d} u)$ and $\tilde{M}(\mathrm{~d} s, \mathrm{~d} z, \mathrm{~d} u)$ denote a Gaussian white noise and an independent compensated Poisson random measure, respectively. The model of Li [9] corresponds to solution $\left(X_{t}\right)_{t>0}$ to SDE (1.1) with power rate functions $a_{1}, a_{2}$ and $a_{3}$ of identical power; i.e. $a_{i}(x)=c_{i} x^{r}$ for $r>0$ and $c_{i} \geq 0, i=0,1,2$.

These nonlinear CSBPs allow richer behaviors such as coming down from infinity. Some extinction, extinguishing, explosion and coming down from infinity properties are proved in Li [9]. By analyzing weighted occupation times for spectrally positive Lévy process, asymptotic results on the speeds of coming down from infinity and explosion are obtained in Foucart et al. [2] and in Li and Zhou [8], respectively, for nonlinear CSBP corresponding to solution to SDE (1.1) with rate functions that are mulitplcations of the same nonnegative function $a$; i.e. $a_{i}(x)=c_{i} a(x), i=0,1,2$. Exponential ergodicity for the general continuous-state nonlinear branching processes in Li et al. [11] is studied by Li and Wang [10] using coupling techniques. Long time behaviour of the general continuous-state nonlinear branching processes with catastrophes is studied by Marguet and Smadi [14].

A version of SDE (1.1) with $a_{1} \equiv 0$ and power functions $a_{0}$ and $a_{2}$ is considered earlier in Berestycki et al. [1] where using the Lamperti transform, a necessary and sufficient condition for extinction is obtained and the pathwise uniqueness of solution is studied. Work on the continuous-state logistic branching process can be found in Lambert [5], Le et al. [7] and Le [6].

Using a martingale approach, the extinction, explosion and coming down from infinity behaviors are further discussed in Li et al. [11] and some rather sharp criteria in terms of functions $a_{0}, a_{1}, a_{2}$ and the Poisson random measure are obtained on characterization of different kinds of boundary behaviors for the nonlinear CSBPs as a Markov process. In Example 2.18 of Li et al. [11] where $a_{0}, a_{1}$ and $a_{2}$ are taken to be power functions and $\tilde{M}$ is taken to be an $\alpha$-stable Poison random measure with index $\alpha \in(1,2)$. The above criteria are further expressed in terms of the coefficients and the powers of functions $a_{i}$ and the stable index $\alpha$. But for the critical cases, where the coefficients, the powers and the index $\alpha$ satisfy certain linear equations, the martingale approach fails and the corresponding boundary classification remains an open problem (see Remark 2.3).

The main goal of this paper is to identify the exact boundary behaviors in the above mentioned critical cases for the solution $\left(X_{t}\right)_{t \geq 0}$ to (1.1). For this purpose, we adapt the Foster-Lyapunov approach and select logarithm type test functions to obtain two new conditions under which the nonlinear CSBP never becomes extinct and never explodes, respectively. Similarly, for the boundary at infinity, we also find a Foster-Lyapunov condition with which we can show that an interesting phase transition occurs between coming down from infinity and staying infinite for different choices of coefficients and powers of the power functions and differential values of the stable index $\alpha$.

The rest of the paper is arranged as follows. We introduce the generalized CSBPs with nonlinear branching in more details and present the main theorem in Section 2. The proofs of preliminary results on Foster-Lyapunov criteria and the main theorem are deferred to Section 3.

\section{Main results}

Let $U$ be a Borel set on $(0, \infty)$. Given $\sigma$-finite measure $\mu$ on $(0, \infty)$ such that

$\left(z \wedge z^{2}\right) \mu(\mathrm{d} z)$ and $(1 \vee \ln (1+z)) \mu(\mathrm{d} z)$ are finite measures on $U$ and $(0, \infty) \backslash U$, respectively, 
we consider the following SDE that is a modification of (1.1):

$$
\begin{aligned}
X_{t}= & x+\int_{0}^{t} a_{0}\left(X_{s}\right) \mathrm{d} s+\int_{0}^{t} \int_{0}^{a_{1}\left(X_{s}\right)} W(\mathrm{~d} s, \mathrm{~d} u) \\
& +\int_{0}^{t} \int_{U} \int_{0}^{a_{2}\left(X_{s-}\right)} z \tilde{M}(\mathrm{~d} s, \mathrm{~d} z, \mathrm{~d} u)+\int_{0}^{t} \int_{(0, \infty) \backslash U} \int_{0}^{a_{3}\left(X_{s-}\right)} z M(\mathrm{~d} s, \mathrm{~d} z, \mathrm{~d} u),(2 .)
\end{aligned}
$$

where $x>0, a_{0}$ and $a_{1}, a_{2}, a_{3} \geq 0$ are Borel functions on $[0, \infty), W(\mathrm{~d} s, \mathrm{~d} u)$ is a Gaussian white noise with density $\mathrm{d} s \mathrm{~d} u$ and $M(\mathrm{~d} s, \mathrm{~d} z, \mathrm{~d} u)$ denotes a Poisson random measure on $(0, \infty)^{3}$ with density $\mathrm{d} s \mu(\mathrm{d} z) \mathrm{d} u$. Let $\tilde{M}(\mathrm{~d} s, \mathrm{~d} z, \mathrm{~d} u)$ be the compensated Poisson random measure of $M(\mathrm{~d} s, \mathrm{~d} z, \mathrm{~d} u)$. We assume that $W(\mathrm{~d} s, \mathrm{~d} u)$ and $\tilde{M}(\mathrm{~d} s, \mathrm{~d} z, \mathrm{~d} u)$ are independent of each other. A similar SDE (2.1) is considered in Li et al. [11] under the assumption that $U=(0, \infty)$.

We only consider the solution of (2.1) before the minimum of their first times of hitting zero and reaching infinity (that is minimum of $\tau_{0}^{-}$and $\tau_{\infty}^{+}$, which will be given in the following), respectively, i.e. both zero and infinity are absorbing states for the solution. See Section 2 of Li et al. [11] for more details. By the same argument as Theorem 3.1 in Li et al. [11], we can show that SDE (2.1) has a pathwise unique solution if functions $a_{0}, a_{1}, a_{2}, a_{3}$ are locally Lipschitz on $(0, \infty)$. The main purpose of this paper is to investigate the extinction, explosion and coming down from infinity behaviors, and the uniqueness of solution to SDE (2.1) is not required.

Throughout this paper we always assume that $a_{0}, a_{1}, a_{2}, a_{3}$ are bounded on any bounded interval of $[0, \infty)$ and that process $\left(X_{t}\right)_{t \geq 0}$ is defined on filtered probability space $\left(\Omega, \mathscr{F}, \mathscr{F}_{t}, \mathbf{P}\right)$ which satisfies the usual hypotheses. We use $\mathbf{P}_{x}$ to denote the law of a process started at $x$, and denote by $\mathbf{E}_{x}$ the associated expectation. For $a, b>0$ we define the first passage times

$$
\tau_{a}^{-}:=\inf \left\{t>0: X_{t} \leq a\right\}, \quad \tau_{b}^{+}:=\inf \left\{t>0: X_{t} \geq b\right\}
$$

and

$$
\tau_{0}^{-}:=\inf \left\{t>0: X_{t}=0\right\}, \quad \tau_{\infty}^{+}:=\lim _{n \rightarrow \infty} \tau_{n}^{+}
$$

with the convention $\inf \emptyset=\infty$. Let $C^{2}((0, \infty))$ denote the space of twice continuously differentiable functions on $(0, \infty)$.

We next introduce several auxiliary functions. For $u>0$ let

$$
\begin{aligned}
\phi(u):= & -a_{0}(u) u^{-1}+\frac{1}{2} a_{1}(u) u^{-2}+a_{2}(u) \int_{U} z^{2} \mu(\mathrm{d} z) \int_{0}^{1}(u+v z)^{-2}(1-v) \mathrm{d} v \\
& -a_{3}(u) \int_{(0, \infty) \backslash U} z \mu(\mathrm{d} z) \int_{0}^{1}(u+v z)^{-1} \mathrm{~d} v .
\end{aligned}
$$

For $\rho, z>0$ and $u>3$ let

$$
K_{\rho}(u, z):=\left(\frac{\ln (u+z)}{\ln u}\right)^{-\rho}+\rho \frac{\ln (u+z)}{\ln u}-(\rho+1)>0
$$

and

$$
H_{\rho}(u):=\frac{1}{2} a_{1}(u) u^{-2}+a_{2}(u) \int_{U} K_{\rho}(u, z) \mu(\mathrm{d} z)+a_{3}(u) \int_{(0, \infty) \backslash U} K_{\rho}(u, z) \mu(\mathrm{d} z),
$$

which will be used in the proofs for Theorem 2.1 (iii) and (iv).

Process $\left(X_{t}\right)_{t \geq 0}$ becomes extinct if $\tau_{0}^{-}<\infty$; it explodes if $\tau_{\infty}^{+}<\infty$; it stays infinite if $\lim _{x \rightarrow \infty} \mathbf{P}_{x}\left\{\tau_{a}^{-}<t\right\}=0$ for all $t>0$ and all large $a$; it comes down from infinity if $\lim _{a \rightarrow \infty} \lim _{x \rightarrow \infty} \mathbf{P}_{x}\left\{\tau_{a}^{-}<t\right\}=1$ for all $t>0$.

The following main result provides new criteria on non-extinction, non-explosion, coming down from infinity and staying infinite for the solution to SDE (1.1). 
Theorem 2.1. For the solution $\left(X_{t}\right)_{t \geq 0}$ to (2.1) we have

(i) if $\phi(u) \leq 0$ for all small enough $u>0$, then $\mathbf{P}_{x}\left\{\tau_{0}^{-}=\infty\right\}=1$ for all $x>0$, i.e. there is no extinction;

(ii) if $\phi(u) \geq 0$ for all large enough $u>0$, then $\mathbf{P}_{x}\left\{\tau_{\infty}^{+}=\infty\right\}=1$ for all $x>0$, i.e. there is no explosion;

(iii) if $\phi(u) \leq 0$ for all large enough $u>0$ and

$$
\limsup _{u \rightarrow \infty} H_{\rho}(u)<\infty
$$

for some constant $\rho>0$, then the process $\left(X_{t}\right)_{t \geq 0}$ stays infinite;

(iv) if $\phi(u) \geq 0$ for all large enough $u>0$ and

$$
\lim _{u \rightarrow \infty}(\ln u)^{-\rho-2} H_{\rho}(u)=\infty
$$

for some constant $\rho>0$, then the process $\left(X_{t}\right)_{t \geq 0}$ does not explode and it comes down from infinity.

Remark 2.2. Since the process $\left(X_{t}\right)_{t \geq 0}$ does not explode and comes down from infinity under the assumptions of Theorem 2.1 (iv), it can be extended to a Feller process defined on state space $[0, \infty]$ with $\infty$ as its entrance boundary given that it is Feller on $[0, \infty)$; see Theorem 2.2 of Foucart et al. [2]. If $\left(X_{t}\right)_{t \geq 0}$ further solves SDE (1.1), then the conditions on functions $a_{0}, a_{1}, a_{2}$ for process $\left(X_{t}\right)_{t \geq 0}$ to be Feller on $[0, \infty)$ can also be found in Foucart et al. [2].

Until the end of this section we focus on the special case that $U=(0, \infty), a_{0}, a_{1}, a_{2}$ are power functions and $\mu(\mathrm{d} z)$ is an $\alpha$-stable measure, that is

$$
a_{i}(u)=b_{i} u^{r_{i}} \text { for } i=0,1,2 \text { with } r_{0}, r_{1}, r_{2} \geq 0, b_{1}, b_{2} \geq 0, b_{0}>0
$$

and

$$
\mu(\mathrm{d} z)=\frac{\alpha(\alpha-1)}{\Gamma(2-\alpha)} 1_{\{z>0\}} z^{-1-\alpha} \mathrm{d} z \text { for Gamma function } \Gamma \text { and } 1<\alpha<2 .
$$

By the properties of Gamma function we have

$$
\int_{0}^{\infty} z^{2} \mu(\mathrm{d} z) \int_{0}^{1}(u+v z)^{-2}(1-v) \mathrm{d} v=\Gamma(\alpha) u^{-\alpha}
$$

and then

$$
\phi(u)=-b_{0} u^{r_{0}-1}+\frac{1}{2} b_{1} u^{r_{1}-2}+\Gamma(\alpha) b_{2} u^{r_{2}-\alpha}, \quad u>0 .
$$

We further estimate

$$
H_{\rho}(u)=\frac{1}{2} a_{1}(u) u^{-2}+a_{2}(u) \int_{0}^{\infty} K_{\rho}(u, z) \mu(\mathrm{d} z)
$$

for which we first estimate $\int_{0}^{\infty} K_{\rho}(u, z) \mu(\mathrm{d} z)$. Note that for $y>0$ and $f(y):=y^{-\rho}+\rho y-$ $(\rho+1)$, by Taylor's formula we have

$$
\begin{aligned}
f(y) & =f(1+y-1)=f(1+y-1)-f(1)-(y-1) f^{\prime}(1) \\
& =(y-1)^{2} \int_{0}^{1} f^{\prime \prime}(1+v(y-1))(1-v) \mathrm{d} v .
\end{aligned}
$$

Then by a change of variable,

$$
\int_{0}^{\infty} K_{\rho}(u, z) \mu(\mathrm{d} z)
$$




$$
\begin{aligned}
& =\rho(\rho+1) \int_{0}^{\infty} \mu(\mathrm{d} z) \int_{0}^{1}\left(\frac{\ln (u+v z)}{\ln u}-1\right)^{2}\left(1+\frac{v \ln (u+v z)}{\ln u}-v\right)^{-\rho-2}(1-v) \mathrm{d} v \\
& =\rho(\rho+1) u^{-\alpha} \int_{0}^{\infty} \mu(\mathrm{d} z) \int_{0}^{1}\left(\frac{\ln (1+v z)}{\ln u}\right)^{2}\left(1+\frac{v \ln (1+v z)}{\ln u}\right)^{-\rho-2}(1-v) \mathrm{d} v .
\end{aligned}
$$

Observe that $\ln (1+z) \leq C(z \wedge \sqrt{z})$ for all $z>0$ and some constant $C>0$, which implies that for $u>3$,

$$
\begin{aligned}
\int_{0}^{\infty} K_{\rho}(u, z) \mu(\mathrm{d} z) & \leq \rho(\rho+1) u^{-\alpha}(\ln u)^{-2} \int_{0}^{\infty}(\ln (1+z))^{2} \mu(\mathrm{d} z) \\
& \leq C^{2} \rho(\rho+1) u^{-\alpha}(\ln u)^{-2} \int_{0}^{\infty}\left(z \wedge z^{2}\right) \mu(\mathrm{d} z)
\end{aligned}
$$

Moreover, it is elementary to see that

$$
\begin{aligned}
& \int_{0}^{\infty} K_{\rho}(u, z) \mu(\mathrm{d} z) \\
\geq & \rho(\rho+1) u^{-\alpha}(\ln u)^{-2}\left(1+\frac{\ln 3}{\ln u}\right)^{-\rho-2} \int_{1}^{2}(\ln (3 / 2))^{2} \mu(\mathrm{d} z) \int_{1 / 2}^{1}(1-v) \mathrm{d} v .
\end{aligned}
$$

Remark 2.3. In Section 2.5 of Li et al. [11], the exact conditions are found for the above mentioned model with polynomial rate functions to exhibit extinction/non-extinction, explosion/non-explosion and coming-down-from-infinity/staying-infinite behaviors, respectively, except for the critical case that

$$
b_{0}=\frac{b_{1}}{2}+\Gamma(\alpha) b_{2}>0, r_{1}=r_{0}+1 \text { when } b_{1}>0 \text { and } r_{2}=r_{0}+\alpha-1 \text { when } b_{2}>0 .
$$

Observe that in this critical case $\phi(u)=L(\ln )(u)=0$, where the operator $L$, to be defined in (3.2), denotes the generator of process $X$. This inspires us to choose logarithm type test functions for the main proofs.

As the main goal of this paper, applying Theorem 2.1 together with (2.4)-(2.5), we provide an answer to this open problem.

Corollary 2.4. Suppose that (2.2) and (2.3) hold with $b_{0}=\frac{b_{1}}{2}+\Gamma(\alpha) b_{2}>0, r_{1}=r_{0}+1$ when $b_{1}>0$ and $r_{2}=r_{0}+\alpha-1$ when $b_{2}>0$. Then $\phi(u)=0$ for all $u>0$, and we have for all $x>0$,

$$
\mathbf{P}_{x}\left\{\tau_{0}^{-}=\infty\right\}=1 \quad \text { and } \quad \mathbf{P}_{x}\left\{\tau_{\infty}^{+}=\infty\right\}=1 .
$$

Moreover, process $\left(X_{t}\right)_{t \geq 0}$ stays infinite if both $r_{1} \leq 2$ (when $b_{1}>0$ ) and $r_{2} \leq \alpha$ (when $b_{2}>0$ ), and it comes down from infinity if either $r_{1}>2$ (when $b_{1}>0$ ) or $r_{2}>\alpha$ (when $\left.b_{2}>0\right)$.

Remark 2.5. Note that in the critical cases, there is an interesting phase transition between coming down from infinity and staying infinite. Intuitively, in these cases the process comes down from infinity if the fluctuations caused by the Brownian motion and the Poisson random measure are relatively large and stays infinite otherwise.

Combining Corollary 2.4 and Example 2.18 of Subsection 2.5 in Li et al. [11], we recover the necessary and sufficient condition on the extinction of solution to the SDE of Berestycki et al. [1]; see Theorem 1.1 there.

\section{Proofs}

Before presenting the proof of Theorem 2.1 we first prove some preliminary FosterLyapunov criteria. Suppose that $g \in C^{2}((0, \infty))$ satisfies

$$
\sup _{z \geq 1, u \geq v}\left[\left|g^{\prime}(u)\right|+\left|g^{\prime \prime}(u)\right|+|g(u+z)-g(u)| / \ln (1+z)\right]<\infty
$$


for all $v>0$. For $u>0$, put

$$
\begin{aligned}
L g(u):= & a_{0}(u) g^{\prime}(u)+\frac{1}{2} a_{1}(u) g^{\prime \prime}(u)+a_{2}(u) \int_{U}\left[g(u+z)-g(u)-z g^{\prime}(u)\right] \mu(\mathrm{d} z) \\
& +a_{3}(u) \int_{(0, \infty) \backslash U}[g(u+z)-g(u)] \mu(\mathrm{d} z) \\
= & a_{0}(u) g^{\prime}(u)+\frac{1}{2} a_{1}(u) g^{\prime \prime}(u)+a_{2}(u) \int_{U} z^{2} \mu(\mathrm{d} z) \int_{0}^{1} g^{\prime \prime}(u+z v)(1-v) \mathrm{d} v \\
& +a_{3}(u) \int_{(0, \infty) \backslash U} z \mu(\mathrm{d} z) \int_{0}^{1} g^{\prime}(u+z v) \mathrm{d} v
\end{aligned}
$$

by Taylor's formula. By Itô's formula,

$$
\begin{aligned}
g\left(X_{t}\right)= & g(x)+\int_{0}^{t} L g\left(X_{s}\right) \mathrm{d} s+\int_{0}^{t} \int_{U} \int_{0}^{a_{2}\left(X_{s-}\right)}\left[g\left(X_{s-}+z\right)-g\left(X_{s-}\right)\right] \tilde{M}(\mathrm{~d} s, \mathrm{~d} z, \mathrm{~d} u) \\
& +\int_{0}^{t} \int_{(0, \infty) \backslash U}^{a_{3}\left(X_{s-}\right)}\left[g\left(X_{s-}+z\right)-g\left(X_{s-}\right)\right] \tilde{M}(\mathrm{~d} s, \mathrm{~d} z, \mathrm{~d} u)
\end{aligned}
$$

For $b>a>0$ let $\gamma_{a, b}:=\tau_{a}^{-} \wedge \tau_{b}^{+}$and

$$
M_{t}^{g}:=g\left(X_{t}\right)-g(x)-\int_{0}^{t} L g\left(X_{s}\right) \mathrm{d} s .
$$

Then under condition (3.1),

$$
t \mapsto M_{t \wedge \gamma_{a}, b}^{g} \quad \text { is a martingale }
$$

for all $b>a>0$.

Lemma 3.1. Given $0<a<x<b<\infty$, for any function $g \in C^{2}((a, b))$ satisfying (3.1) and constant $d_{a, b}>0$ satisfying

$$
\operatorname{Lg}(u) \leq d_{a, b} g(u), \quad u \in(a, b),
$$

we have

$$
\mathbf{E}_{x}\left[g\left(X_{t \wedge \gamma_{a, b}}\right)\right] \leq g(x) \mathrm{e}^{d_{a, b} t}, \quad t \geq 0 .
$$

Proof. It follows from (3.3) that

$$
\mathbf{E}_{x}\left[g\left(X_{t \wedge \gamma_{a, b}}\right)\right]=g(x)+\mathbf{E}_{x}\left[\int_{0}^{t \wedge \gamma_{a, b}} L g\left(X_{s}\right) \mathrm{d} s\right] \leq g(x)+d_{a, b} \int_{0}^{t} \mathbf{E}_{x}\left[g\left(X_{s \wedge \gamma_{a, b}}\right)\right] \mathrm{d} s .
$$

By Gronwall's lemma,

$$
\mathbf{E}_{x}\left[g\left(X_{t \wedge \gamma_{a, b}}\right)\right] \leq g(x) \mathrm{e}^{d_{a, b} t},
$$

which ends the proof.

Lemma 3.2. Let $\left(X_{t}\right)_{t \geq 0}$ be the solution to $S D E$ (2.1).

(i) For any fixed $b>0$, if there exists a function $g \in C^{2}((0, \infty))$ strictly positive on $(0, b]$ satisfying (3.1) and $\lim _{u \rightarrow 0} g(u)=\infty$, and there is a constant $d(b)>0$ such that $L g(u) \leq d(b) g(u)$ for all $0<u<b$, then $\mathbf{P}_{x}\left\{\tau_{0}^{-} \geq \tau_{b}^{+}\right\}=1$ for all $0<x<b$.

(ii) For any fixed $a>0$, if there exists a function $g \in C^{2}((0, \infty))$ strictly positive on $[a, \infty)$ satisfying (3.1) and $\lim _{u \rightarrow \infty} g(u)=\infty$, and there is a constant $d(a)>0$ such that $\operatorname{Lg}(u) \leq d(a) g(u)$ for all $u>a$, then $\mathbf{P}_{x}\left\{\tau_{\infty}^{+} \geq \tau_{a}^{-}\right\}=1$ for all $x>a$. 
(iii) If there exists a function $g \in C^{2}((0, \infty))$ strictly positive on $[u, \infty)$ for all large $u$ satisfying (3.1) and $\lim _{u \rightarrow \infty} g(u)=0$, and for any large $a>0$, there is a constant $d(a)>0$ such that $L g(u) \leq d(a) g(u)$ for all $u>a$, then $\left(X_{t}\right)_{t \geq 0}$ stays infinite.

Proof. We apply Lemma 3.1 for the proofs.

For part (i), (3.4) holds for all $0<a<b$ and with $d_{a, b}$ replaced by $d(b)$. Then using Fatou's lemma we have

$$
\mathbf{E}_{x}\left[\liminf _{a \rightarrow 0} g\left(X_{t \wedge \tau_{a}^{-} \wedge \tau_{b}^{+}}\right)\right] \leq \liminf _{a \rightarrow 0} \mathbf{E}_{x}\left[g\left(X_{t \wedge \gamma_{a, b}}\right)\right] \leq g(x) \mathbf{e}^{d(b) t} .
$$

Since $\lim _{u \rightarrow 0} g(u)=\infty$, then $\mathbf{P}_{x}\left\{\tau_{0}^{-}>t \wedge \tau_{b}^{+}\right\}=1$ for all $t, b>0$. Letting $t \rightarrow \infty$ we obtain $\mathbf{P}_{x}\left\{\tau_{0}^{-} \geq \tau_{b}^{+}\right\}=1$, which gives the first assertion.

For part (ii), (3.4) holds for all $b>a$ and with $d_{a, b}$ replaced by $d(a)$. Then using Fatou's lemma again we obtain

$$
\mathbf{E}_{x}\left[\liminf _{b \rightarrow \infty} g\left(X_{t \wedge \tau_{a}^{-} \wedge \tau_{b}^{+}}\right)\right] \leq \liminf _{b \rightarrow \infty} \mathbf{E}_{x}\left[g\left(X_{t \wedge \gamma_{a, b}}\right)\right] \leq g(x) \mathbf{e}^{d(a) t} .
$$

Since $\lim _{u \rightarrow \infty} g(u)=\infty$, then $\mathbf{P}_{x}\left\{\tau_{\infty}^{+}>t \wedge \tau_{a}^{-}\right\}=1$ for all $t>0$. Letting $t \rightarrow \infty$ we get $\mathbf{P}_{x}\left\{\tau_{\infty}^{+} \geq \tau_{a}^{-}\right\}=1$, which implies the second assertion.

For part (iii), given any large $a>0$, (3.4) holds for all $b>a$ and with $d_{a, b}$ replaced by $d(a)$ again. We can also get

$$
\begin{aligned}
\mathbf{E}_{x}\left[g\left(X_{\tau_{a}^{-}}\right) 1_{\left\{\tau_{a}^{-}<t \wedge \tau_{\infty}^{+}\right\}}\right] & \leq \liminf _{b \rightarrow \infty} \mathbf{E}_{x}\left[g\left(X_{\tau_{a}^{-}}\right) 1_{\left\{\tau_{a}^{-}<t \wedge \tau_{b}^{+}\right\}}\right] \\
& \leq \liminf _{b \rightarrow \infty} \mathbf{E}_{x}\left[g\left(X_{t \wedge \gamma_{a, b}}\right)\right] \leq g(x) \mathbf{e}^{d(a) t},
\end{aligned}
$$

which implies

$$
g(a) \mathbf{P}_{x}\left\{\tau_{a}^{-}<t \wedge \tau_{\infty}^{+}\right\} \leq g(x) \mathrm{e}^{d(a) t} .
$$

Since $\lim _{u \rightarrow \infty} g(u)=0$, then for all $t, a>0$,

$$
\lim _{x \rightarrow \infty} \mathbf{P}_{x}\left\{\tau_{a}^{-}<t \wedge \tau_{\infty}^{+}\right\}=0 .
$$

Observe that $\left\{\tau_{a}^{-} \geq \tau_{\infty}^{+}\right\} \subset\left\{\tau_{a}^{-}=\infty\right\}$. Then combining (3.5) we have

$$
\lim _{x \rightarrow \infty} \mathbf{P}_{x}\left\{\tau_{a}^{-}<t\right\} \leq \lim _{x \rightarrow \infty} \mathbf{P}_{x}\left\{\tau_{a}^{-}<t \wedge \tau_{\infty}^{+}\right\}+\lim _{x \rightarrow \infty} \mathbf{P}_{x}\left\{\tau_{a}^{-}<t, \tau_{\infty}^{+} \leq \tau_{a}^{-}\right\}=0
$$

for all $t>0$. Then the process stays infinite.

The next lemma provides a condition that associates the probability of coming down from infinity with the probability of non-explosion. Its proof is a modification of Proposition 2.2 in Ren et al. [15].

Lemma 3.3. Suppose that there exist a function $g(u) \in C^{2}((0, \infty))$ bounded and strictly positive for all large $u$, satisfying (3.1) and $\lim \sup _{u \rightarrow \infty} g(u)>0$, and a strictly positive function $d$ on $(0, \infty)$ such that

$$
L g(u) \geq d(a) g(u) \text { for all } u \geq a \text { and } \lim _{a \rightarrow \infty} d(a)=\infty .
$$

Then for any $t>0$

$$
\lim _{a \rightarrow \infty} \lim _{x \rightarrow \infty} \mathbf{P}_{x}\left\{\tau_{a}^{-}<t\right\} \geq \liminf _{x \rightarrow \infty} \mathbf{P}_{x}\left\{\tau_{\infty}^{+}=\infty\right\} .
$$

Consequently, process $\left(X_{t}\right)_{t \geq 0}$ comes down from infinity if there is no explosion. 
Proof. The proof is a modification of that of Proposition 2.2 in Ren et al. [15]. We present the details for completeness. By (3.3), for all large $a<b$

$$
\mathbf{E}_{x}\left[g\left(X_{t \wedge \gamma_{a, b}}\right)\right]=g(x)+\mathbf{E}_{x}\left[\int_{0}^{t \wedge \gamma_{a, b}} L g\left(X_{s}\right) \mathrm{d} s\right]=g(x)+\int_{0}^{t} \mathbf{E}_{x}\left[L g\left(X_{s}\right) 1_{\left\{s \leq \gamma_{a, b}\right\}}\right] \mathrm{d} s
$$

and then by integration by parts,

$$
\begin{aligned}
& \mathbf{E}_{x}\left[g\left(X_{t \wedge \gamma_{a, b}}\right)\right] \mathrm{e}^{-d(a) t} \\
= & g(x)+\int_{0}^{t} \mathbf{E}_{x}\left[g\left(X_{s \wedge \gamma_{a, b}}\right)\right] \mathrm{d}\left(\mathrm{e}^{-d(a) s}\right)+\int_{0}^{t} \mathrm{e}^{-d(a) s} \mathrm{~d}\left(\mathbf{E}_{x}\left[g\left(X_{s \wedge \gamma_{a, b}}\right)\right]\right) \\
= & g(x)-d(a) \int_{0}^{t} \mathbf{E}_{x}\left[g\left(X_{s \wedge \gamma_{a, b}}\right) \mathrm{e}^{-d(a) s}\right] \mathrm{d} s+\int_{0}^{t} \mathrm{e}^{-d(a) s} \mathbf{E}_{x}\left[L g\left(X_{s}\right) 1_{\left\{s \leq \gamma_{a, b}\right\}}\right] \mathrm{d} s \\
\geq & g(x)-d(a) \int_{0}^{t} \mathbf{E}_{x}\left[g\left(X_{s \wedge \gamma_{a, b}}\right)\right] \mathrm{e}^{-d(a) s} \mathrm{~d} s+d(a) \int_{0}^{t} \mathrm{e}^{-d(a) s} \mathbf{E}_{x}\left[g\left(X_{s}\right) 1_{\left\{s \leq \gamma_{a, b}\right\}}\right] \mathrm{d} s,
\end{aligned}
$$

which implies that

$$
g(x) \leq \mathbf{E}_{x}\left[g\left(X_{t \wedge \gamma_{a, b}}\right) \mathrm{e}^{-d(a) t}\right]+d(a) \mathbf{E}_{x}\left[\int_{0}^{t} g\left(X_{\gamma_{a, b}}\right) \mathrm{e}^{-d(a) s} 1_{\left\{s>\gamma_{a, b}\right\}} \mathrm{d} s\right] .
$$

Letting $t \rightarrow \infty$ in the above inequality and using the dominated convergence we obtain

$$
g(x) \leq d(a) \mathbf{E}_{x}\left[g\left(X_{\gamma_{a, b}}\right) \int_{\gamma_{a, b}}^{\infty} \mathrm{e}^{-d(a) s} \mathrm{~d} s\right]=\mathbf{E}_{x}\left[g\left(X_{\gamma_{a, b}}\right) \mathrm{e}^{-d(a) \gamma_{a, b}}\right] .
$$

It follows that

$$
\begin{aligned}
g(x) & \leq \mathbf{E}_{x}\left[\lim _{b \rightarrow \infty} g\left(X_{\gamma_{a, b}}\right) \mathrm{e}^{-\left(\tau_{a}^{-} \wedge \tau_{\infty}^{+}\right) d(a)}\left(1_{\left\{\tau_{\infty}^{+}<\tau_{a}^{-}\right\}}+1_{\left\{\tau_{a}^{-}<t, \tau_{a}^{-} \leq \tau_{\infty}^{+}\right\}}+1_{\left\{t \leq \tau_{a}^{-} \leq \tau_{\infty}^{+}\right\}}\right)\right] \\
& \leq \limsup _{u \rightarrow \infty} g(u) \mathbf{P}_{x}\left\{\tau_{\infty}^{+}<\infty\right\}+g(a) \mathbf{P}_{x}\left\{\tau_{a}^{-}<t, \tau_{a}^{-} \leq \tau_{\infty}^{+}\right\}+g(a) \mathrm{e}^{-d(a) t} \\
& \leq \limsup _{u \rightarrow \infty} g(u)\left(1-\mathbf{P}_{x}\left\{\tau_{\infty}^{+}=\infty\right\}\right)+g(a) \mathbf{P}_{x}\left\{\tau_{a}^{-}<t\right\}+g(a) \mathrm{e}^{-d(a) t} .
\end{aligned}
$$

Letting $x \rightarrow \infty$ first,

$$
\begin{aligned}
\limsup _{x \rightarrow \infty} g(x) \leq & \limsup _{u \rightarrow \infty} g(u) \limsup _{x \rightarrow \infty}\left(1-\mathbf{P}_{x}\left\{\tau_{\infty}^{+}=\infty\right\}\right) \\
& +g(a) \lim _{x \rightarrow \infty} \mathbf{P}_{x}\left\{\tau_{a}^{-}<t\right\}+g(a) \mathbf{e}^{-d(a) t}
\end{aligned}
$$

Then letting $a \rightarrow \infty$, by the conditions in the lemma we have

$$
\begin{aligned}
\limsup _{x \rightarrow \infty} g(x) \leq & \limsup _{u \rightarrow \infty} g(u)\left(1-\liminf _{x \rightarrow \infty} \mathbf{P}_{x}\left\{\tau_{\infty}^{+}=\infty\right\}\right) \\
& +\limsup _{a \rightarrow \infty} g(a) \limsup _{a \rightarrow \infty} \lim _{x \rightarrow \infty} \mathbf{P}_{x}\left\{\tau_{a}^{-}<t\right\} .
\end{aligned}
$$

Observing that $\lim _{x \rightarrow \infty} \mathbf{P}_{x}\left\{\tau_{a}^{-}<t\right\}$ is increasing in $a$, the desired inequality then follows from the above inequality.

We are now ready to show the proofs of the main results.

Proof of Theorem 2.1. (i) Suppose that there is a constant $0<c_{1}<1$ so that $\phi(u) \leq 0$ for all $0<u<c_{1}$. For $n \geq 1$ let $g_{n}(u)=1+\ln n+\ln u^{-1}$. Then $g_{n}(u)>0$ for $0<u \leq n$ and $L g_{n}(u)=\phi(u)$ by (3.2). Thus $L g_{n}(u) \leq 0$ for $0<u<c_{1}$. Since $a_{0}, a_{1}, a_{2}, a_{3}$ are bounded on $\left[c_{1}, n\right], L g_{n}$ is bounded on $\left[c_{1}, n\right]$. Now using Lemma 3.2(i) we obtain $\mathbf{P}_{x}\left\{\tau_{0}^{-} \geq \tau_{n}^{+}\right\}=1$ for all $0<x<n$. Since the process is defined before the first time of hitting zero or explosion, $\mathbf{P}_{x}\left\{\tau_{0}^{-}=\infty\right.$ or $\left.\tau_{\infty}^{+}=\infty\right\}=1$. Letting $n \rightarrow \infty$ we prove the assertion.

(ii) Suppose that there is a constant $c_{2}>1$ so that $\phi(u) \geq 0$ for all $u>c_{2}$. Let $g_{n}(u)=\ln u+\ln n+1$ for $n \geq 1$. Then $g_{n}(u) \geq 1$ for $u \geq n^{-1}$. It follows from (3.2) that 
$L g_{n}(u)=-\phi(u)$ for all $u \geq n^{-1}$. Then for all $n \geq 1, L g_{n}(u) \leq 0$ for all $u \geq c_{2}$ and $L g_{n}$ is bounded on $\left[n^{-1}, c_{2}\right]$, which gives $\mathbf{P}_{x}\left\{\tau_{\infty}^{+}>\tau_{1 / n}^{-}\right\}=1$ for all $x>n^{-1}$ by Lemma 3.2(ii). Letting $n \rightarrow \infty$ we have $\mathbf{P}_{x}\left\{\tau_{\infty}^{+}>\tau_{0}^{-}\right\}=1$ for all $x>0$. The assertion for (ii) then follows from the definition of the solution to SDE (2.1).

(iii) Suppose that there exist constants $c_{3}>3$ and $c_{4}>0$ so that $\phi(u) \leq 0$ and $H_{\rho}(u) \leq c_{4}$ for all $u>c_{3}$. Let $g \in C^{2}((0, \infty))$ be a strictly positive function with $g(u)=(\ln u)^{-\rho}$ for $\rho>0$ and $u>3$.

Then for $u>3$,

$$
\begin{aligned}
g(u+z)-g(u) & =-\rho(\ln u)^{-\rho-1}[\ln (u+z)-\ln u]+(\ln u)^{-\rho} K_{\rho}(u, z) \\
& =-\rho(\ln u)^{-\rho-1} z \int_{0}^{1}(u+v z)^{-1} \mathrm{~d} v+(\ln u)^{-\rho} K_{\rho}(u, z)
\end{aligned}
$$

and

$$
g^{\prime}(u)=-\rho(\ln u)^{-\rho-1} u^{-1}, g^{\prime \prime}(u)=\rho(\ln u)^{-\rho-1} u^{-2}+\rho(\rho+1)(\ln u)^{-\rho-2} u^{-2} .
$$

Consequently, for all $u>3$ and $z>0$,

$$
\begin{aligned}
g(u+z)-g(u)-z g^{\prime}(u) & =-\rho(\ln u)^{-\rho-1}\left[\ln (u+z)-\ln u-z u^{-1}\right]+(\ln u)^{-\rho} K_{\rho}(u, z) \\
& =\rho(\ln u)^{-\rho-1} z^{2} \int_{0}^{1}(u+z v)^{-2}(1-v) \mathrm{d} v+(\ln u)^{-\rho} K_{\rho}(u, z) .
\end{aligned}
$$

It follows that

$$
\begin{aligned}
L g(u)= & \rho(\ln u)^{-\rho-1} \phi(u)+\frac{1}{2} \rho(\rho+1)(\ln u)^{-2} a_{1}(u) u^{-2} g(u) \\
& +g(u) a_{2}(u) \int_{U} K_{\rho}(u, z) \mu(\mathrm{d} z)+g(u) a_{3}(u) \int_{(0, \infty) \backslash U} K_{\rho}(u, z) \mu(\mathrm{d} z) \\
\leq & \rho(\ln u)^{-\rho-1} \phi(u)+[\rho(\rho+1)+1] g(u) H_{\rho}(u), \quad u>3 .
\end{aligned}
$$

Then $L g(u) \leq c_{4}[\rho(\rho+1)+1] g(u)$ for all $u>c_{3}$. Thus, $\left(X_{t}\right)_{t \geq 0}$ stays infinite for all $x>0$ by Lemma 3.2(iii).

(iv) Let $g \in C^{2}((0, \infty))$ be a bounded and strictly positive function with $g(u)=$ $1+(\ln u)^{-\rho}$ for $\rho>0$ and $u>3$. It follows from the argument in (iii) that for $u>3$,

$$
\begin{aligned}
L g(u)= & \rho(\ln u)^{-\rho-1} \phi(u)+\frac{1}{2} \rho(\rho+1)(\ln u)^{-\rho-2} a_{1}(u) u^{-2} \\
& +(\ln u)^{-\rho} a_{2}(u) \int_{U} K_{\rho}(u, z) \mu(\mathrm{d} z)+(\ln u)^{-\rho} a_{3}(u) \int_{(0, \infty) \backslash U} K_{\rho}(u, z) \mu(\mathrm{d} z) \\
\geq & \rho(\ln u)^{-\rho-1} \phi(u)+(\rho \wedge 1)(\ln u)^{-\rho-2} H_{\rho}(u) .
\end{aligned}
$$

Then we can conclude the proof by the assumptions for this part together with Theorem 2.1(ii) and Lemma 3.3.

\section{References}

[1] Berestycki, J., Döring, L., Mytnik, L. and Zambotti, L.: Hitting properties and non-uniqueness for sdes driven by stable processes. Stochastic Process. Appl. 125, (2015), 918-940. MR3303962

[2] Foucart, C., Li, P.-S. and Zhou, X.: Time-changed spectrally positive Lévy processes started from infinity. To appear in Bernoulli, arXiv:1901.10689.

[3] Foucart, C., Li, P.-S. and Zhou, X.: On the entrance at infinity of Feller processes with no negative jumps. Statist. Probab. Lett., 165, (2020), 108859. MR-4118938

[4] Kyprianou, A. E.: Introductory Lectures on Fluctuations of Lévy Processes with Applications. Springer, Berlin, Heidelberg, 2012. MR-2250061 
[5] Lambert, A.: The branching process with logistic growth. Ann. Appl. Probab, 15, (2005), 1506-1535. MR-2134113

[6] Le, V.: Branching process with interaction. Ph.D. thesis, Reading Aix-Marseille University, 2014.

[7] Le, V., Pardoux, E. and Wakolbinger, A.: “Trees under attack": A Ray-Knight representation on Feller's branching diffusion with logistic growth. Probab. Theory Related Fields, 155, (2013), 583-619. MR-3034788

[8] Li, B. and Zhou, X.: On the explosion of a class of continuous-state nonlinear branching processes, arXiv:1910.05914.

[9] Li, P.-S.: A continuous-state polynomial branching process. Stochastic Process. Appl., 129, (2019), 2941-2967. MR-3980150

[10] Li, P.-S. and Wang, J.: Exponential ergodicity for general continuous-state nonlinear branching processes. Electron. J. Probab., 25, (2020), 1-25. MR-4161135

[11] Li, P.-S., Yang, X. and Zhou, X.: A general continuous-state nonlinear branching process. Ann. Appl. Probab., 29, (2019), 2523-2555. MR-3983343

[12] Li, Z.: Measure-Valued Branching Markov Process. Springer, Berlin, Heidelberg, 2011. MR-2760602

[13] Li, Z.: Continuous-state branching processes with immigration, arXiv:1901.03521.

[14] Marguet, A. and Smadi, C.: Long time behaviour of continuous-state nonlinear branching processes with catastrophes, arXiv:1908.11592v2.

[15] Ren, Y., Xiong, J., Yang, X. and Zhou, X.: On the extinction-extinguishing dichotomy for a stochastic Lotka-Volterra type population dynamical system, arXiv:1912.10182.

Acknowledgments. The authors thank an anonymous referee for helpful comments. This work was supported by Key research project for North Minzu University (No. 2019KJ28), NSFC (Nos. 11772002, 11771018, 11731012 and 12061004), NSF of Ningxia (No. 2020AAC03230), Major research project for North Minzu University (No. ZDZX201902), the Construction Project of First-Class Disciplines in Ningxia Higher Education (No. NXYLXK2017B09) and NSERC (RGPIN-2016-06704). 